\title{
Metastatic ovarian cancer spreading into mammary ducts mimicking an in situ component of primary breast cancer: a case report
}

Yurina Maeshima ${ }^{1,2}$, Tomo Osako ${ }^{1, *^{*}}$ (D, Hidetomo Morizono ${ }^{2}$, Mayu Yunokawa ${ }^{4}$, Yumi Miyagi ${ }^{2}$, Mari Kikuchi ${ }^{5}$, Takayuki Ueno ${ }^{2}$, Shinji Ohno² and Futoshi Akiyama'

\begin{abstract}
Background: Accurate diagnosis of metastatic tumors in the breast is crucial because the therapeutic approach is essentially different from primary tumors. A key morphological feature of metastatic tumors is their lack of an in situ carcinoma component. Here, we present a unique case of metastatic ovarian carcinoma spreading into mammary ducts and mimicked an in situ component of primary carcinoma. To our knowledge, this is the second case (and the first adult case) confirming the in situ-mimicking growth pattern of a metastatic tumor using immunohistochemistry.

Case presentation: A 69-year-old Japanese woman was found to have a breast mass with microcalcifications. She had a known history of ovarian mixed serous and endocervical-type mucinous (seromucinous) carcinoma. Needle biopsy specimen of the breast tumor revealed adenocarcinoma displaying an in situ-looking tubular architecture in addition to invasive micropapillary and papillary architectures with psammoma bodies. From these morphological features, metastatic serous carcinoma and invasive micropapillary carcinoma of breast origin were both suspected. In immunohistochemistry, the cancer cells were immunoreactive for WT1, PAX8, and CA125, and negative for GATA3, mammaglobin, and gross cystic disease fluid protein-15. Therefore, the breast tumor was diagnosed to be metastatic ovarian serous carcinoma. The in situ-looking architecture showed the same immunophenotype, but was surrounded by myoepithelium confirmed by immunohistochemistry (e.g. p63, cytokeratin 14, CD10). Thus, the histogenesis of the in situ-like tubular foci was could be explained by the spread of metastatic ovarian cancer cells into existing mammary ducts.

Conclusion: Metastatic tumors may spread into mammary duct units and mimic an in situ carcinoma component of primary breast cancer. This in situ-mimicking growth pattern can be a potential pitfall in establishing a correct diagnosis of metastasis to the breast. A panel of breast-related and extramammary organ/tumor-specific immunohistochemical markers may be helpful in distinguishing metastatic tumors from primary tumors.
\end{abstract}

Keywords: Breast metastasis, Ovarian serous carcinoma, Invasive micropapillary carcinoma, In situ component, Case report

*Correspondence: tomo.osako@jfcr.or.jp

${ }^{1}$ Division of Pathology, Cancer Institute of Japanese Foundation for Cancer Research, 3-8-31, Ariake, Koto-ku, Tokyo 135-8550, Japan

Full list of author information is available at the end of the article

\section{Introduction}

Breast cancer is the most common malignancy in women worldwide. However, metastases to the breast from extramammary solid tumors are rare and account for only $0.2-0.9 \%$ of all breast malignancies $[1-3]$. The most original author(s) and the source, provide a link to the Creative Commons licence, and indicate if changes were made. The images or other third party material in this article are included in the article's Creative Commons licence, unless indicated otherwise in a credit line to the material. If material is not included in the article's Creative Commons licence and your intended use is not permitted by statutory regulation or exceeds the permitted use, you will need to obtain permission directly from the copyright holder. To view a copy of this licence, visit http://creativecommons.org/licenses/by/4.0/. The Creative Commons Public Domain Dedication waiver (http://creativeco mmons.org/publicdomain/zero/1.0/) applies to the data made available in this article, unless otherwise stated in a credit line to the data. 
common primary tumors metastasizing to the breast vary depending on the specific patient population studied [4], but malignant melanoma, lung carcinoma, ovarian carcinoma, gastrointestinal carcinoma, and sarcoma are repeatedly reported [4-7].

Accurate diagnosis of metastatic tumors in the breast is crucial because their staging, treatment and prognosis are essentially different from primary breast tumors [6]. One of the key morphological features for the diagnosis of metastatic tumors is their lack of an in situ (intraductal and/or intralobular) carcinoma component $[3,5,7]$. The presence of an in situ component strongly supports the diagnosis of primary carcinoma. However, in this case report, we present a unique case of metastatic ovarian carcinoma spreading into mammary ducts, which mimicked an in situ component of primary breast carcinoma.

\section{Case presentation \\ Clinical summary}

A 69-year-old Japanese woman was found to have a right breast mass with calcifications and pleural nodules on computed tomography for post-treatment surveillance of ovarian cancer (Fig. 1a). This patient had undergone debulking surgery and chemotherapy (carboplatin plus paclitaxel) for stage IIIc ovarian cancer 9 years before, and she had received additional chemotherapy (carboplatin plus paclitaxel) for bilateral axillary lymph node
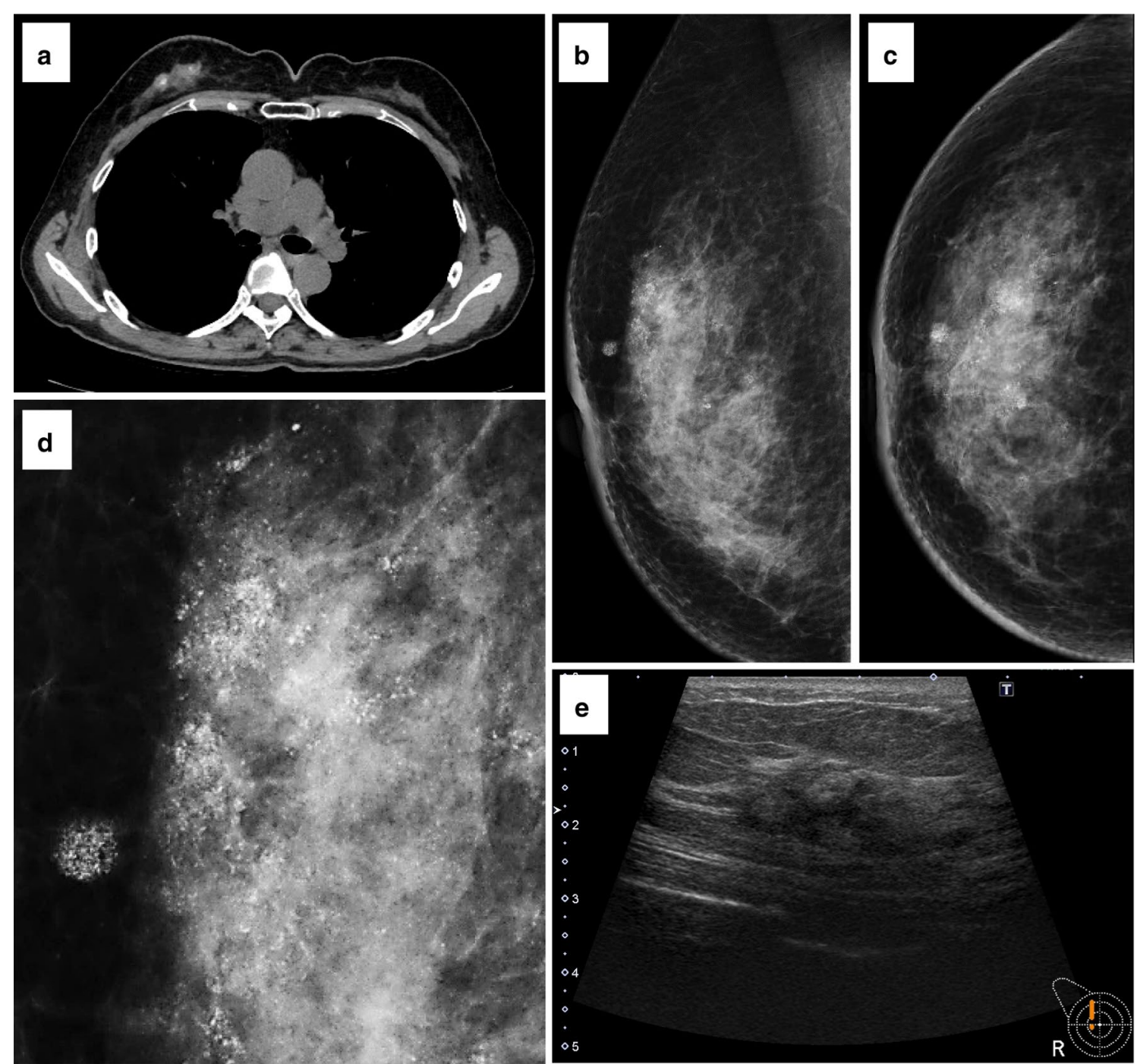

Fig. 1 Radiological images of the metastatic ovarian serous carcinoma in the breast. Plain chest computed tomography (a); mediolateral-oblique and craniocaudal views of the mammography $(\mathbf{b}, \mathbf{c})$ and magnified image of the mediolateral-oblique view (d); and ultrasonography (e) 
metastasis 4 years before. Then, she had been followed up every 3 months.

On physical examination, a $33 \times 30-\mathrm{mm}$ induration was palpable in the upper-outer quadrant of the patient's right breast. Mammography showed segmental distribution of the amorphous microcalcifications associated with a focal asymmetric density in the upper-outer area of the breast (Fig. 1b-d). Ultrasonography showed a $33 \times 33 \times 16-\mathrm{mm}$ irregular hypoechoic area with high echo spots and indistinct margins (Fig. 1e). From these images, ductal carcinoma in situ or invasive ductal carcinoma with a predominant intraductal component was primarily suspected, but metastatic ovarian cancer could not be excluded considering her clinical history. The patient underwent vacuum-assisted needle biopsy for the breast lesion. After the biopsy, she was treated with chemotherapy (carboplatin plus gemcitabine followed by carboplatin plus doxorubicin) for 2 years. At the time of this report ( 2 and half years after the biopsy), she receives best supportive care for metastatic ovarian cancer and myelodysplastic syndrome.

\section{Pathological findings of previous ovarian tumor}

Macroscopic examination of the surgical specimens revealed a $65 \times 55 \times 45$ - $\mathrm{mm}$, lobulated, whitish-yellow, solid mass in the right ovary; an $8-\mathrm{mm}$ mass on the surface of the left ovary; and multiple disseminated tumors, up to $16 \mathrm{~mm}$ in size, in the greater omentum.

Microscopically, the right ovarian tumor displayed a complex branching papillary architecture (Fig. 2a). The epithelium lining the papillae was stratified and was composed of endocervical-type mucinous epithelium (Fig. 2b) and serous epithelium (Fig. 2c). There were multiple invasive cancer foci in the stroma composed of endocervical-type mucinous epithelium displaying tubular architecture (Fig. 2d) and serous epithelium displaying micropapillary architecture with psammoma bodies (Fig. 2e). Thus, the tumor was diagnosed to be seromucinous carcinoma (mixed endocervical-type mucinous and low-grade serous carcinoma) associated with seromucinous borderline tumor. The immunohistochemical analysis supported the diagnosis (Table 1).

The left ovary was also diagnosed with seromucinous carcinoma with seromucinous borderline tumor. In the omentum, there were multiple disseminations of serous carcinoma accompanied with psammoma bodies and desmoplastic stroma (Fig. 2f).

\section{Pathological findings of breast tumor}

Microscopic examination of the needle biopsy specimen revealed invasive adenocarcinoma displaying micropapillary and papillary architectures in the breast stroma (Fig. 3a, b). The cancer cells displayed intermediate nuclear atypia and low mitotic activity. Psammoma bodies were frequently observed. In addition, a small number of cancer nests showed a tubular architecture surrounded by myoepithelium, which appeared to be an in situ carcinoma (Fig. 3a, 3c, 3e). On the hematoxylin-and-eosinstained slides, metastatic serous carcinoma was primarily suspected due to the morphological similarities to the previous ovarian carcinoma, but invasive micropapillary carcinoma of the breast origin could not be ruled out considering the in situ-like foci.

In immunohistochemistry, the cancer cells were immunoreactive for WT1 (Fig. 3d), PAX8, and CA125, and negative for GATA3, mammaglobin, and gross cystic disease fluid protein-15 (GCDFP15) (Table 1). Therefore, the breast tumor was diagnosed to be metastatic ovarian serous carcinoma.

Regarding the in situ-like tubular foci, the cancer cells lining the tubule and papillae showed the aforementioned immunophenotype (e.g. positive WT1, Fig. 3f), but the tubular foci were surrounded by myoepithelium which was immunoreactive for myoepithelial markers [p63 (Fig. 3g), cytokeratin 14 (Fig. 3h), CD10 and calponin], and negative for endothelial markers (podoplanin and CD31). Thus, the histogenesis of the in situ-like tubular foci may be explained by the spread of metastatic ovarian cancer cells into existing mammary ducts.

\section{Discussion}

We present a unique case of ovarian carcinoma metastasizing to the breast and spreading into mammary ducts, which mimicked an in situ component of primary breast carcinoma. One case report of metastatic pancreatic tumor in a child firstly confirmed this in situ-mimicking growth pattern by immunohistochemistry [8]. Two other reports briefly mentioned this growth pattern of metastatic tumors, but appropriate immunohistochemical stains to prove intraductal growth were not performed $[2,9]$. Thus, to our knowledge, this is the second case (and the first adult case) confirming the growth pattern by immunohistochemistry. This in situ-mimicking growth pattern can be a potential pitfall for establishing a correct diagnosis of metastasis. The same growth pattern, aside from the metastatic tumor, was recently reported in a soft tissue tumor arising in the breast [10].

Histologically correct and type-specific diagnosis of tumors metastasizing to the breast is vital to ensure appropriate management. However, because of their rarity, it is sometimes difficult for pathologists to make the accurate diagnosis. The following four points can be given as diagnostic clues for metastatic tumors $[2-5,7$, 11, 12]: (1) clinical history of extramammary malignancy, (2) unusual morphology for primary breast cancer, (3) 


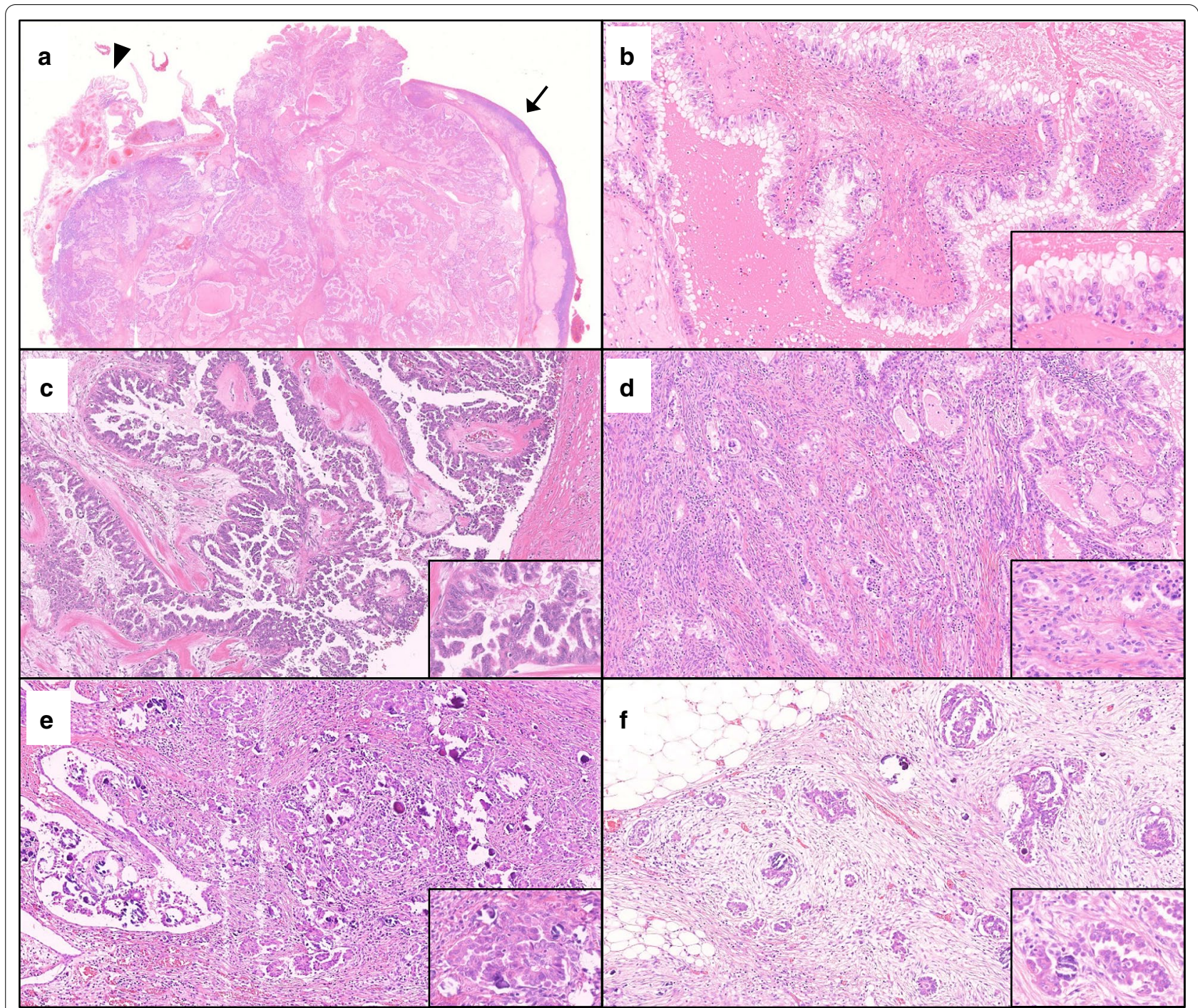

Fig. 2 Pathological images of the primary ovarian tumor and omental dissemination. Hematoxylin-and-eosin-stainined images of the primary tumor: loupe view (a magnification $\times 5$; arrow, ovary; arrow head, Fallopian tube), intermediate-magnification views of seromucinous borderline tumor (mixed endocervical-type mucinous tumor $[\mathbf{b}, \times 100]$ and serous tumor $[\mathbf{c}, \times 100]$ ) and seromucinous carcinoma (mixed endocervical-type mucinous carcinoma $[\mathbf{d}, \times 100]$ and low-grade serous carcinoma $[\mathbf{e}, \times 100]$. Hematoxylin-and-eosin-stainined images of omental dissemination of low-grade serous carcinoma $(\mathbf{f}, \times 100)$.

absence of an in situ component, and (4) lack of breastrelated immunophenotype.

Clinical history of extramammary cancer is essential in making a diagnosis of metastasis to the breast $[3,5,11]$. Almost all breast cancer cases that pathologists diagnose in daily practice are primary cancers. Thus, suspicion of metastatic tumors may sometimes be raised only after clinical history is provided. Comparison of mammary and extramammary tumors is important in this situation.

The diagnosis of metastatic tumors is easier when the tumor has an unusual appearance for a breast primary lesion or a typical morphology of its primary site of origin
[12]. Two-thirds of metastases to the breast have distinctive histological features, raising the possibility of the diagnosis [3]. In remaining cases, however, the histological appearance is similar to a primary mammary tumor, and the clinical history and other information are important to establish the correct diagnosis for these cases.

The presence of an in situ carcinoma component is pathognomonic of primary breast carcinoma. On the contrary, the absence of an in situ component supports the diagnosis of a metastatic tumor to the breast $[3,5$, 7]. However, in situ-like atypical ductal proliferations are reported in metastatic tumors in the breast, and 
Table 1. Antibodies for immunostaining and results for the present case.

\begin{tabular}{|c|c|c|c|c|c|c|}
\hline \multirow[t]{3}{*}{ Antibody } & \multirow[t]{3}{*}{ Clone } & \multirow[t]{3}{*}{ Source } & \multirow[t]{3}{*}{ Dilution } & \multicolumn{3}{|l|}{ Immunoreactivity } \\
\hline & & & & \multicolumn{2}{|l|}{ Ovarian carcinoma } & \multirow{2}{*}{ Breast tumor } \\
\hline & & & & $\begin{array}{l}\text { Mucinous, endocervical } \\
\text { type (seromucinous) }\end{array}$ & Serous, low grade & \\
\hline WT1 & WT49 & Leica & $1 / 30$ & $(+)$ & $(+++)$ & $(+++)$ \\
\hline PAX8 & $\mathrm{BC} 12$ & Nichirei & RTU & $(+)$ & $(+++)$ & $(+++)$ \\
\hline CA125 & M11 & Dako & $1 / 50$ & $(+++)$ & $(+++)$ & $(+++)$ \\
\hline p53 & DO-7 & Dako & $1 / 2000$ & wild type & wild type & wild type \\
\hline$E R$ & SP1 & Ventana & RTU & $(+++)$ & $(+++)$ & $(+++)$ \\
\hline $\mathrm{PgR}$ & 1E2 & Ventana & RTU & $(++)$ & $(+)$ & $(-)$ \\
\hline GATA3 & HG3-31 & Santa Cruz & $1 / 50$ & $(-)$ & $(-)$ & $(-)$ \\
\hline Mammaglobin & 304-1A5 & Dako & $1 / 500$ & $(-)$ & $(-)$ & $(-)$ \\
\hline GCDFP15 & D6 & BioLegend & $1 / 500$ & $(-)$ & $(-)$ & $(-)$ \\
\hline HER2 & $4 \mathrm{~B} 5$ & Ventana & RTU & $(-)$ & $(-)$ & $(-)$ \\
\hline Ki67 & MIB1 & Dako & $1 / 50$ & $15 \%$ & $15 \%$ & $15 \%$ \\
\hline
\end{tabular}

ER estrogen receptor, PgR progesterone receptor, GCDFP15 gross cystic disease fluid protein-15, HER2 human epidermal growth factor-2, RTU ready to use, (+) focal and weak staining, $(++)$ intermediate staining, $(+++)$ diffuse and strong staining

pathologists should be careful not to regard this in situlike structure as true in situ carcinoma and exclude the possibility of metastatic tumor. The in situ-mimicking lesions in metastatic tumors can be classified into three types based on histogenesis: (a) lymphovascular emboli from metastatic tumors [2, 11], (b) metastatic tumors spreading into existing mammary duct units [2, 8, 9], and (c) true in situ carcinoma or atypical ductal/lobular hyperplasia of breast origin coexisting with metastatic tumors $[9,12]$. The immunohistochemical panel mentioned below can be useful for the differential diagnosis (Table 2).

Immunohistochemistry plays a major role in the accurate diagnosis of metastatic tumor in the breast. A panel of breast-related markers (e.g., GATA3, mammaglobin, GCDFP15, and SOX10) is helpful to rule out a metastasis [5]. In addition, a panel of extramammary organ/ tumor-specific markers can be used to delineate the likely primary site of metastasis [5]. In addition, myoepithelial markers (e.g. p63, cytokeratin 14, and calponin) and endothelial markers (e.g., podoplanin and CD31) may be useful for the differential diagnosis of in situ-like architecture in the metastatic tumors $[4,5,11]$.

Serous carcinoma is the most common type of ovarian tumor metastasizing to the breast [13]. Metastatic serous carcinoma can resemble invasive micropapillary carcinoma of the breast, and psammomatous calcifications can be seen in both $[3,14,15]$. One study reported that approximately $24 \%$ of metastatic serous carcinomas in the breast were initially interpreted as primary carcinomas [14]. In fact, invasive micropapillary carcinoma was originally designated "pseudopapillary (serous-like) carcinoma" because of its resemblance to serous carcinoma of Müllerian origin [15]. A key morphological finding for the differential diagnosis may be the presence or absence of a fibrovascular core in the papillary/micropapillary structure. Invasive micropapillary carcinoma mainly shows micropapillary (pseudopapillary) architecture without fibrovascular cores [15], while serous carcinoma often displays (macro-) papillary structure with fibrovascular cores in addition to their micropapillary architecture [16]. A panel of breast-related and Müllerian duct/serous tumorspecific immunohistochemical markers (e.g. WT1 and PAX8) can be helpful in differentiating tumors of ovarian from breast origin. However, estrogen and progesterone receptors are not helpful because both tumors can be positive for these [13].

In the present case, immunohistochemistry played a critical role in establishing an accurate diagnosis of the metastatic tumor and in elucidating the histogenesis of its in situ-mimicking architecture. Although the breast tumor was histologically similar to the previous ovarian carcinoma, invasive micropapillary carcinoma of breast origin could not be ruled out because the in situ-like component surrounded by myoepithelium was present. Based on the positive Müllerian/serous markers and negative breast-related markers, the breast tumor and the in situ-like component was diagnosed as metastatic serous carcinoma. The histogenesis of the in situ-like architecture could be due to spread of the metastatic tumor into existing mammary ducts, since myoepithelium around the in situ-like component was confirmed by myoepithelial immunomarkers. 


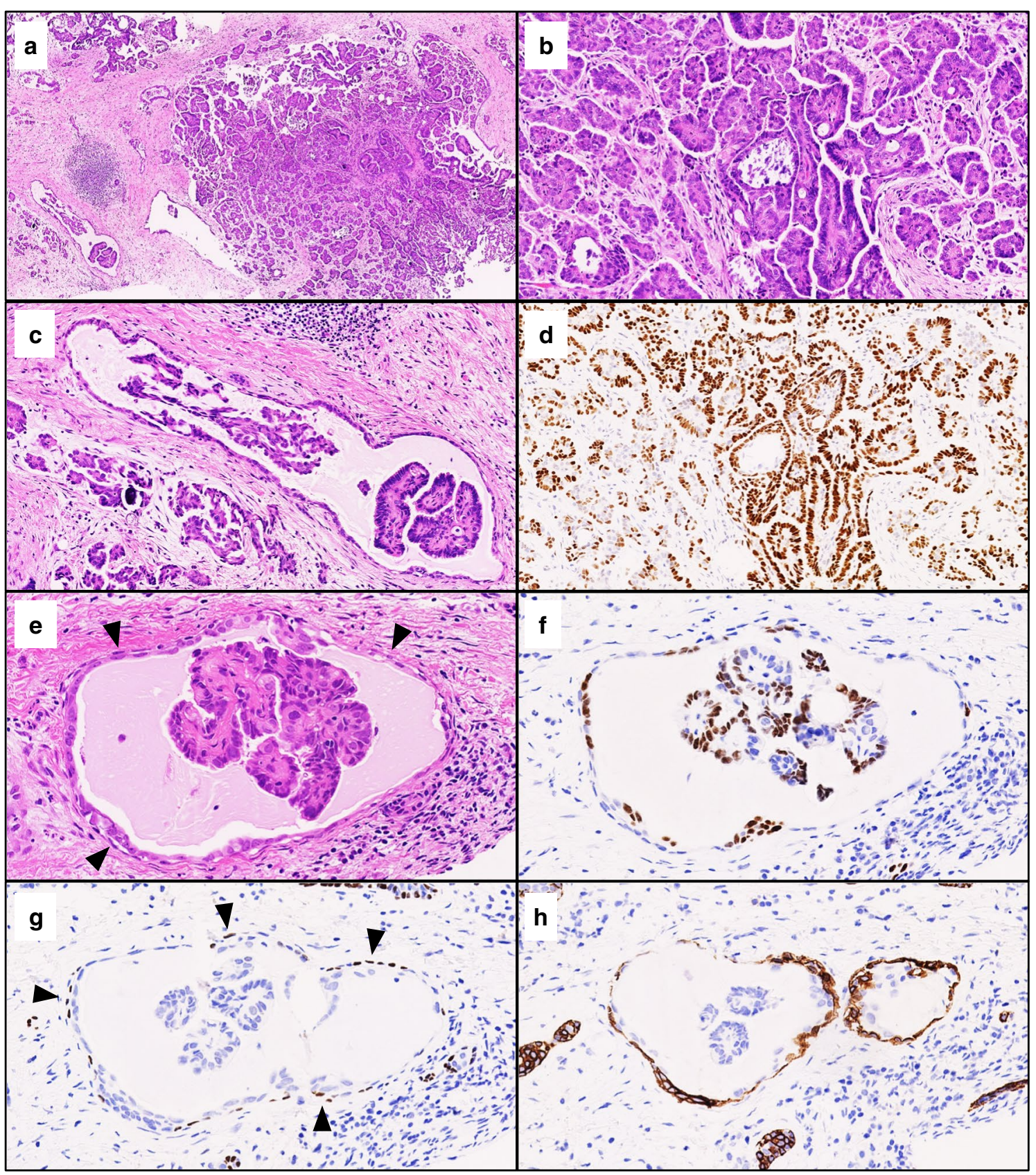

Figure 3. Pathological images of the metastatic ovarian serous carcinoma in the breast. Hematoxylin-and-eosin-stained images of the tumor: low-magnification view (a, magnification $\times 50$ ), intermediate-magnification views of micropapillary/papillary architectures with psammoma bodies $(\mathbf{b}, \times 200)$ and in situ-looking tubular architecture $(\mathbf{c}, \times 200)$. Immunohistochemical image of GATA3 corresponding to the hematoxylin-and-eosin-stained image $(\mathbf{b})(\mathbf{d}, \times 200)$. Immunohistochemical images of the in situ-looking structure corresponding to the hematoxylin-and-eosin-stained image (e; arrowhead, myoepithelium; magnification × 400):WT1 (f), p63 (g; arrowhead, myoepithelium) and cytoketatin 14 (h).

\section{Conclusions}

We present a unique case of metastatic ovarian carcinoma spreading into mammary ducts, which mimicked an in situ component of primary breast carcinoma. This in situ-mimicking growth pattern of metastatic tumors can be a potential pitfall in establishing a correct diagnosis of metastasis to the breast. A panel of breast-related and extramammary organ/tumor-specific immunohistochemical markers may be helpful in distinguishing metastatic tumors from primary tumors. 
Table 2. Immunohistochemical markers for the differential diagnosis of in situ-mimicking architectures in metastasis to the breast

\begin{tabular}{|c|c|c|c|c|}
\hline & $\begin{array}{l}\text { Extramammary organ/ } \\
\text { tumor-specific }\end{array}$ & Breast-related & Myoepithelial & $\begin{array}{l}\text { Lymphatic } \\
\text { endothelial }\end{array}$ \\
\hline Lymphovascular tumor emboli & $(+)$ & $(-)$ & $(-)$ & $(+)$ \\
\hline Intraductal spread by metastatic tumors ${ }^{a}$ & $(+)$ & $(-)$ & $(+)$ & $(-)$ \\
\hline Coexistence of true in situ carcinoma & $(-)$ & $(+)$ & $(+)$ & $(-)$ \\
\hline
\end{tabular}

a The present case

\section{Abbreviation}

GCDFP15: Gross cystic disease fluid protein-15.

\section{Acknowledgements}

We thank Dr. Akiko Tonooka and Dr. Tomohiro Chiba (Division of Pathology, Cancer Institute of Japanese Foundation for Cancer Research) for their comments on the pathological diagnosis of the ovarian tumor.

\section{Authors' contributions}

YMa and TO collected the data and wrote the manuscript. HM and MY provided the treatments to the patients. YMi and MK reviewed the radiological images. TU and SO supervised treatment decision for the patient. FA made the pathological diagnosis of the tumor. All authors read and approved the final manuscript.

\section{Funding}

Not applicable.

\section{Availability of data and materials}

Not applicable.

\section{Ethics approval and consent to participate}

This study was conducted in accordance with the basic principles of the Declaration of Helsinki.

\section{Consent for publication}

Written informed consent was obtained from the patient for publication of this case report and any accompanying images. A copy of the written consent form is available for review by the Editor-in-Chief of this journal.

\section{Competing interests}

None declared.

\section{Author details}

1 Division of Pathology, Cancer Institute of Japanese Foundation for Cancer Research, 3-8-31, Ariake, Koto-ku, Tokyo 135-8550, Japan. ${ }^{2}$ Department of Surgical Oncology, Breast Oncology Center, Cancer Institute Hospital of Japanese Foundation for Cancer Research, Tokyo, Japan. ${ }^{3}$ Department of Pathology, Cancer Institute Hospital of Japanese Foundation for Cancer Research, Tokyo, Japan. ${ }^{4}$ Department of Gynecologic Oncology, Cancer Institute Hospital of Japanese Foundation for Cancer Research, Tokyo, Japan. ${ }^{5}$ Diagnostic Imaging Center, Cancer Institute Hospital of Japanese Foundation for Cancer Research, Tokyo, Japan.

Received: 22 September 2020 Accepted: 28 December 2020 Published online: 17 February 2021

\section{References}

1. Georgiannos SN, Chin J, Goode AW, Sheaff M. Secondary neoplasms of the breast: a survey of the 20th century. Cancer. 2001;92(9):2259-66.
2. Hajdu SI, Urban JA. Cancers metastatic to the breast. Cancer. 1972;29(6):1691-6.

3. Lee AH. The histological diagnosis of metastases to the breast from extramammary malignancies. J Clin Pathol. 2007;60(12):1333-41.

4. Patel S, Silverman JF, Saad RS, Dabbs DJ. Metastatic tumors in the breast. In: Dabbs DJ, editor. Breast pathology. 2nd ed. Elsevier; 2016.

5. Kulka J, Varga Z. Metastases to the breast. In: Board WCOTE, editor. WHO classification of breast tumours. 5th ed. Lyon: IARC Press; 2019.

6. Williams SA, Ehlers RA 2nd, Hunt KK, Yi M, Kuerer HM, Singletary SE, et al. Metastases to the breast from nonbreast solid neoplasms: presentation and determinants of survival. Cancer. 2007;110(4):731-7.

7. DeLair DF, Corben AD, Catalano JP, Vallejo CE, Brogi E, Tan LK. Non-mammary metastases to the breast and axilla: a study of 85 cases. Mod Pathol. 2013;26(3):343-9.

8. Judson K, Argani P. Intraductal spread by metastatic islet cell tumor (well-differentiated pancreatic endocrine neoplasm) involving the breast of a child, mimicking a primary mammary carcinoma. Am J Surg Pathol. 2006;30(7):912-8.

9. Bacchi CE, Wludarski SC, Ambaye AB, Lamovec J, Salviato T, Falconieri G. Metastatic melanoma presenting as an isolated breast tumor: a study of 20 cases with emphasis on several primary mimickers. Arch Pathol Lab Med. 2013;137(1):41-9.

10. Luangxay T, Osako T, Yonekura R, Sugiura Y, Kikuchi M, Gomi N, et al. Giant cell tumor of soft tissue of the breast: case report with H3F3A mutation analysis and review of the literature. Pathol Res Pract. 2020;216(2):152750.

11. Gupta D, Merino MI, Farhood A, Middleton LP. Metastases to breast simulating ductal carcinoma in situ: report of two cases and review of the literature. Ann Diagn Pathol. 2001;5(1):15-20.

12. Badve S. Other malignant lesions of the breast. In: O'Malley FP, Pinder SE, editors. Breast pathology. Foundation in diagnostic pathology. Elsevier; 2006.

13. Yamasaki H, Saw D, Zdanowitz J, Faltz LL. Ovarian carcinoma metastasis to the breast case report and review of the literature. Am J Surg Pathol. 1993;17(2):193-7.

14. Recine MA, Deavers MT, Middleton LP, Silva EG, Malpica A. Serous carcinoma of the ovary and peritoneum with metastases to the breast and axillary lymph nodes: a potential pitfall. Am J Surg Pathol. 2004;28(12):1646-51.

15. Pettinato G, Manivel CJ, Panico L, Sparano L, Petrella G. Invasive micropapillary carcinoma of the breast: clinicopathologic study of 62 cases of a poorly recognized variant with highly aggressive behavior. Am J Clin Pathol. 2004;121(6):857-66.

16. Serous tumours. In: Kurman RJ, Carcangiu ML, Herrington CS, Young RH, editors. WHO classification of tumours of female reproductive organs. 4th ed. Lyon: IARC Press; 2014

\section{Publisher's Note}

Springer Nature remains neutral with regard to jurisdictional claims in published maps and institutional affiliations. 